\title{
Encephalopathy an Atypical Presentation of Intussusception: A Case Report
}

Venma Mampilly, Sasikumar Manalumukkil Sankaran, Ramaraj Subbiah.'

\begin{abstract}
Background: Intussusception Encephalopathy is a pediatric emergency where a stuporous child presents with or without abdominal symptoms. Neurological manifestations of intussusception are an atypical presentation of this condition. It often misleads clinicians to other differential diagnoses of encephalopathy. The Case: We present you a case of 11-month-old child presented with encephalopathy, acute in onset with underlying intussusception. Conclusion: Intussusception encephalopathy is a pediatric emergency. It should be kept as a differential diagnosis when a child presents with complaints of acute onset of drowsiness with or without abdominal symptoms. Early diagnosis could save grave complications and improve the prognosis
\end{abstract}

Key Words: Metabolic encephalopathy; Intussusception; Pediatric Emergency Medicine; Drowsy (Source: MeSH-NLM).

\section{Introduction}

Intussusception is the commonest cause of bowel obstruction in children under 2 years of age. It occurs in 1.4 per 1000 live births. Intussusception is a rare presentation, being the first case reported by Goetting in the year $1990 .{ }^{2}$ It is characterized by the telescoping of one segment of bowel (intussusceptum) into its neighboring segment (intussuscepiens), situated most commonly near the ileo-cecal valve (ileocolic). ${ }^{3}$ Children suffering from this emergency condition will not show its cardinal symptoms. It is often confused with sepsis until blood and stool cultures result in sterile growth and detected by ultrasonography. Therefore, early diagnosis could prevent the time delay in treatment and improve global prognosis.

The aim of this study is to describe an uncommon presentation of intussusception towards its inclusion in the differential diagnosis when having a child with encephalopathy in clinical practice.

\section{The Case}

A 11-month-old baby girl was brought to pediatric emergency with complaints of excessive drowsiness, vomiting where vomitus was not bilious in nature, decreased feeding and history of intake of stale food in the previous night. The mother described that the child woke up in middle of the afternoon with severe cry and fell into sleep after a few minutes. The child had an episode of blood-stained stools in the next morning. Parents did not mention any past history of exanthematous fever, respiratory infections and gastrointestinal infections. The family didn't suffer from similar complaints. Baby was born at term with no antenatal or postnatal complications. Currently, she is on family diet.

On general examination, child appeared lethargic, drowsy, pale and afebrile. Modified Pediatric Glasgow coma scale was calculated to be $11 / 15$. Vitals were stable with pulse rate-120/min, BP-110/70 mm Hg,

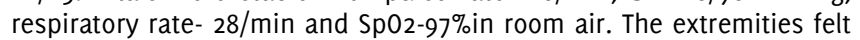
warm. ear, nose and throat examination done and found normal. on abdominal examination a mass was palpable in the epigastrium .She had no features of hepatosplenomegaly and bowel sounds were heard $3 /$ min .On neurological examination, pupils were isochoric and bilateral plantar flexor. There were no other neurological deficits were found. Child showed no signs of meningeal irritation .Other systems were found within normal limits. Anthropometric measurements were assessed and found appropriate for the age. Child was admitted at pediatric intensive care unit for further management (Table 1).

Table 1. Clinical manifestations?

\begin{tabular}{ll}
\hline Symptoms & Signs \\
\hline Altered level of consciousness & $\begin{array}{l}\text { Decreased AVPU scale } \\
\text { Excessive sleepiness }\end{array}$ \\
lethargy & $\begin{array}{l}\text { Sausage shaped mid-abdominal } \\
\text { mass }\end{array}$ \\
Abdominal pain-colicky type & \\
Vomiting & \\
Sudden, loud cry & \\
\hline Legend: AVPU scale: Alert,Verbal,Pain,Unresponsive
\end{tabular}

Routine blood investigations were carried out .A Hemogram showed elevation of total counts of white blood cells of $16.2 \times 10^{9}$ per liter, with Hemoglobin $9.3 \mathrm{~g} / \mathrm{dl}$ and platelet counts of $644 \times 10^{9}$ per liter. Both renal and liver function tests were found within normal limits. Serum electrolytes showed mild hyponatremia with Sodium $(\mathrm{Na}+)$ of 134 , Potassium $(\mathrm{K}+)$ of 4.5 and $\mathrm{HCO}_{3}$ of 18 . Arterial blood gas analysis was done and showed compensated metabolic acidosis (pH: 7.31, Pc02: 38.0, $\mathrm{PO}_{2}: 48.4, \mathrm{HCO}_{3}$ : 18.5). ( $\mathrm{CT}$ brain was taken and detected no features suggestive of intracranial abnormality. Ultrasonography abdomen was done immediately and showed features of ileo -colic intussusceptions (as in Figure 1). A pseudo kidney like bowel related mass lesion of size $8.2 \times 4.2 \times 3.8 \mathrm{~cm}$ formed by herniation of bowel loop along with mesentery into another bowel loop in upper abdomen was suggestive of intussusception with no features of strangulation. Mild ascites was also found in the ultrasound.

Medical student, Kerala University of Health Sciences, Kerala, India.

Professor of Pediatric Surgery, Kerala University of Health Sciences, Kerala, India.

Professor \& Head of the Pediatrics Department, Kerala University of Health Sciences, Kerala, India

About the Author: Venma Mampilly is an intern at the Amala Institute of Medical Sciences, Kerala, India. She has published an article regarding the efficacy of a continuous glucose monitoring system in lowering HbAtc and has also been an author in a study on transgender subjects. She is currently planning to pursue her post-graduation abroad.

Correspondence:

Venma Mampilly

Address: Medical College, P.O, Kerala 680596, India

Email: venma.mampilly@gmail.com
Editor: Mihnea-Alexandru Găman Student Editors: David Avelar Rodriguez \& Ankit Raj Submission: Feb 28, 2020 Acceptance: Jan 5, 2020 Publication: Apr 30, 2020 Process: Peer-reviewed 


\section{Case Report}

Figure 1. Ultrasonographic appearance of the abdomen of the child

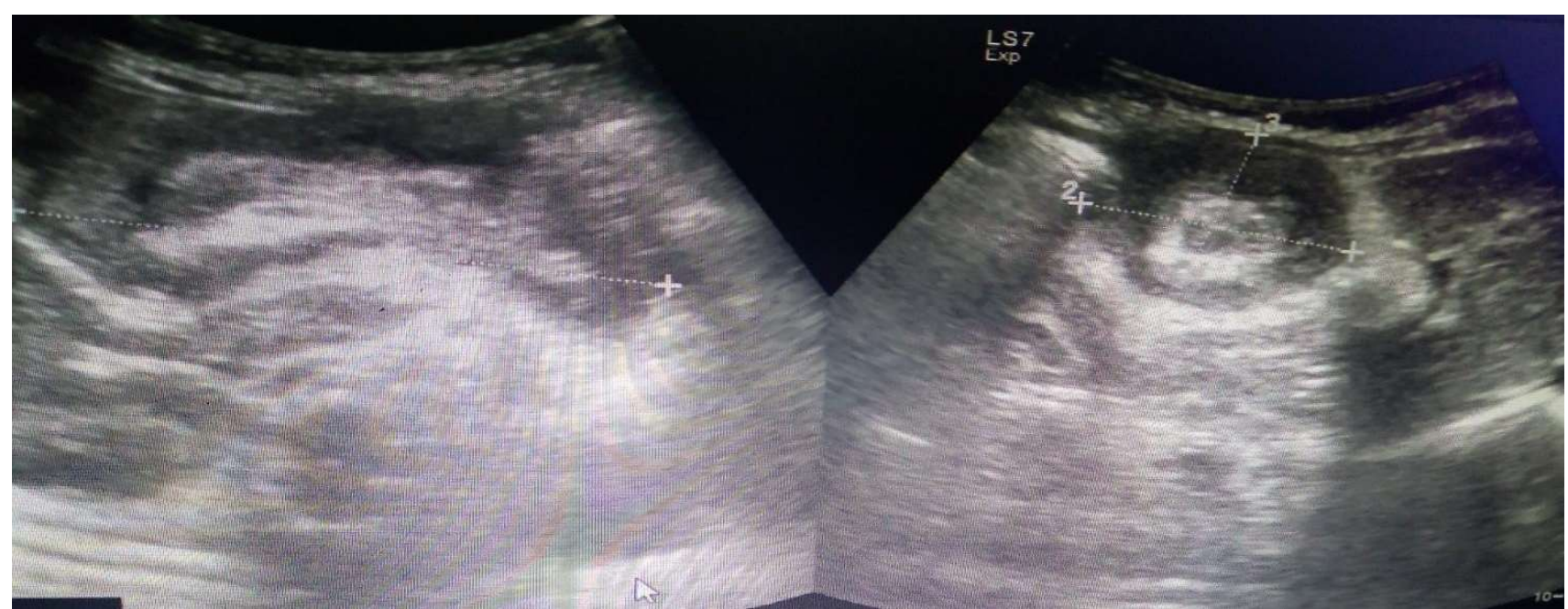

Legend: Target sign of intestinal Intussusception.

Stool and blood were sent for culture and sensitivity to rule out shigellosis and septicemia. Both cultures showed sterile growth. The child was immediately consulted by pediatric surgery and posted for hydrostatic reduction which failed and therefore exploratory laparotomy and reduction was done subsequently (Figure 2). Postoperatively, the child was managed with antibiotics and other supportive measures. Postoperative period was uneventful. The child became more wakeful the next day. Vitals were stable. Surgical site was examined in the following days and there were no features of wound infection seen, hence she was discharged.

\section{Figure 2. Laparoscopic reduction}

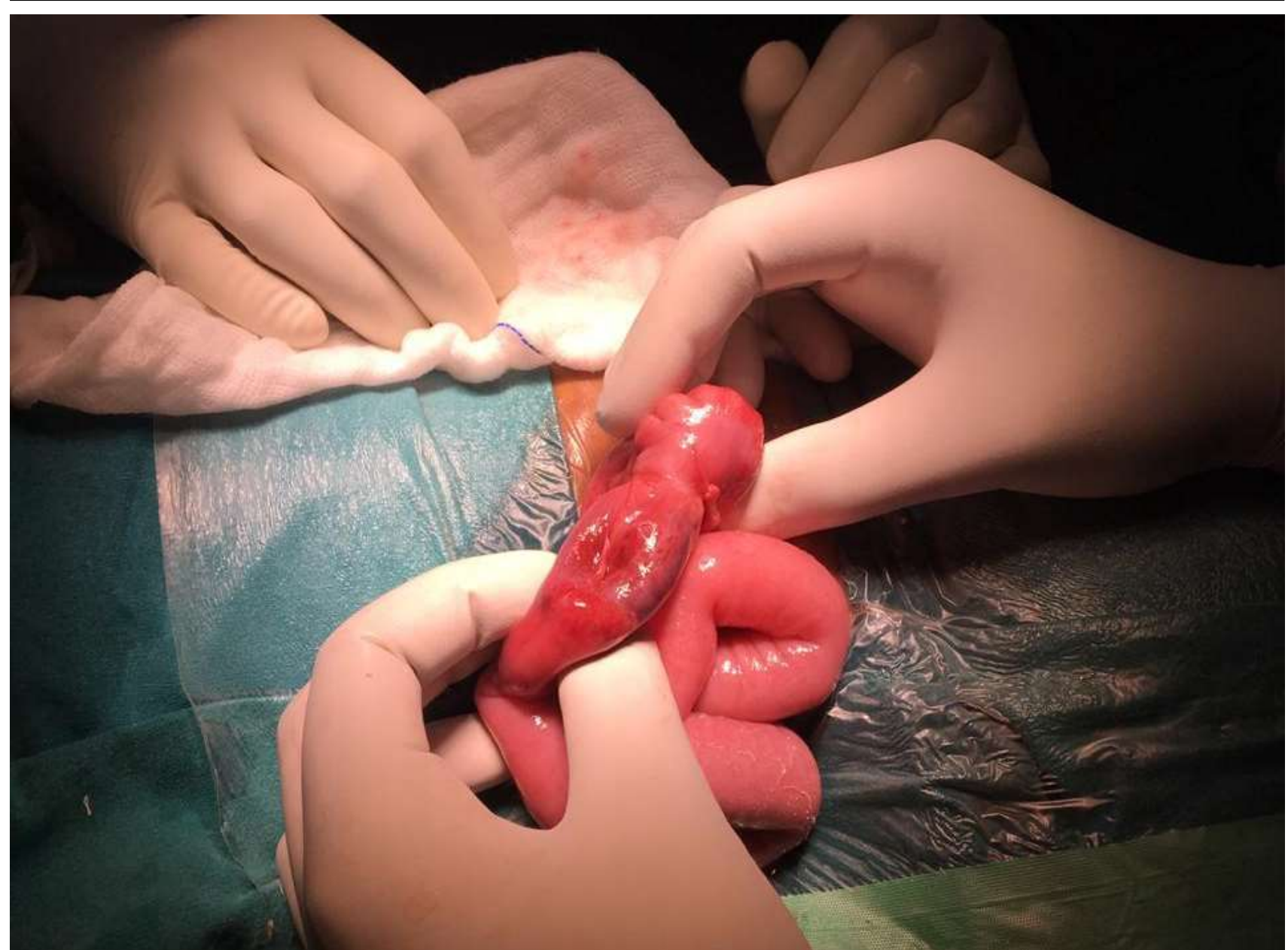

Legend: Telescoping invagination of an intestinal segment into the lumen of a proximal segment (ileocolic). 


\section{Discussion}

Encephalopathy is a generalized disorder of cerebral function that may be acute or chronic, progressive or static. Etiologies include infectious, toxic, metabolic, genetic and ischemic causes. ' The brain which is the most active organ of the human body has a fast-metabolic rate .It requires high level of oxygen to meet this high efficiency. On the other hand, its capacity of energy deposition is very low. When an etiology leading to acute change in consciousness is treated, neurological improvement occurs and the prognosis good. 4

Intussusception could be initiated by introduction of new food, gastrointestinal infection and acute respiratory tract infection. It results in swollen peyer's patches in the terminal ileum. ${ }^{4}$ Intussusception encephalopathy is an atypical neurologic manifestation of intussusception. The neurological symptoms include paroxysmal episodes, global hypotonia, acute weakness and fluctuation of level of consciousness. ${ }^{5}$ Therefore intussusception encephalopathy should be kept as differential diagnosis when children are presented with altered level of consciousness or other neurological symptoms with or without abdominal features. ${ }^{6-7}$

Three possible hypotheses for this encephalopathy have been postulated. First hypothesis is due to the systemic action of toxic metabolites released from ischemic gut that depress the central nervous system as postulated by Singer in his article.9 Another hypothesis proposed for similar manifestation is that a possible endogenous opioid poisoning by massive secretion of endorphins during pain's paroxysm. ${ }^{8}$ This pathophysiology is not clearly understood and a subsequent study demonstrated no difference in plasma beta endorphins levels in patients admitted with intussusception compared to the controls. ${ }^{11}$ Finally, when there is an acute abdominal pathology, it is possible that there will be derangement in the electrolyte and subsequently a metabolic encephalopathy could set in (Figure 3).

Kinnier Wilson coined the term metabolic encephalopathy to describe a clinical state of global cerebral dysfunction induced by systemic stress. ${ }^{11}$ Metabolic encephalopathy can set in due to various mechanisms such as focal or global cerebral edema, alterations in transmitter function, the accumulation of uncleared toxic metabolites, post capillary venule vasogenic edema and energy failure. ${ }^{11}$ In our case , arterial and venous blood analysis shows metabolic acidosis, thereby metabolic encephalopathy may be a possibility in this scenario. This case highlights that one should have a high index of suspicion when a child presents with altered level of consciousness with or without abdominal features suggestive of intussusception.

Figure 3. Hypothetical mechanisms causing Intussusception encephalopathy

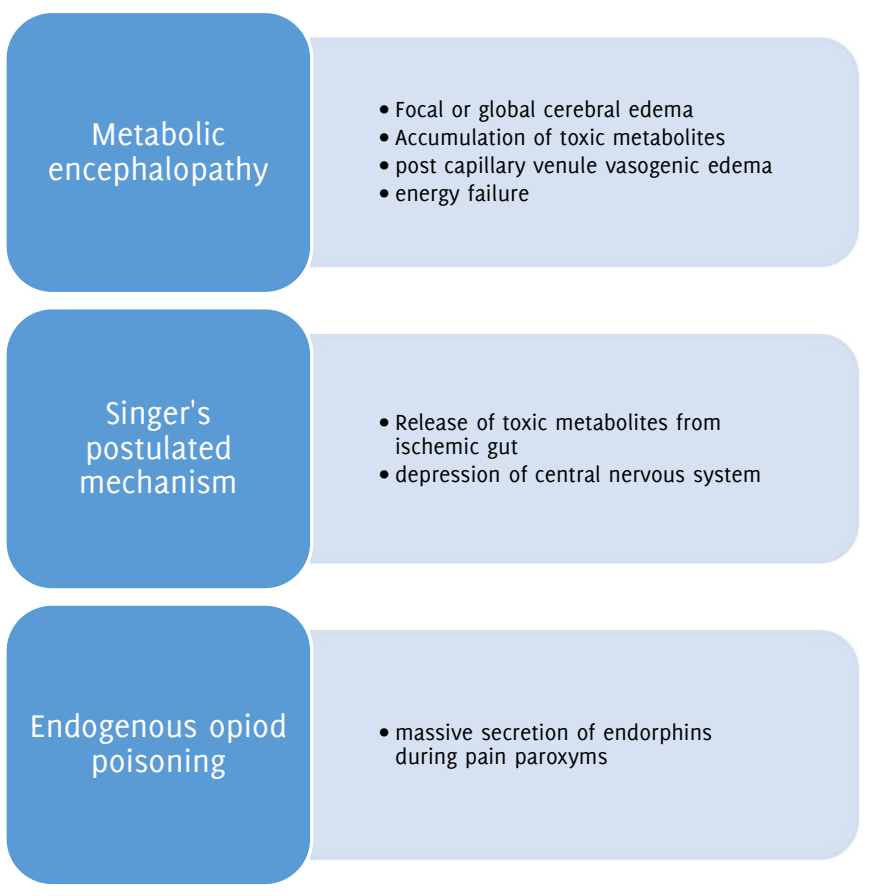




\section{References}

1. Kliegman RM, Behrman RE, Jenson HB, Stanton BM. Nelson textbook of pediatrics. Elsevier Health Sciences; 2007 Aug 15

2. Goetting MG, Tiznado-Garcia E, Bakdash TF. Intussusception encephalopathy: an underrecognized cause of coma in children. Pediatr Neurol. 1990 Nov Dec;6(6):419-21.

3. Dankoff $S$, Puligandla $P$, Beres $A$, Bhanji F. An unusual presentation of smal bowel intussusception. CJEM. 2015 May;17(3):318-21.

4. Aygün F, Aydın PÖ, Emre Ş, Uzunoğlu SŞ, Saltık S, Çam H. A rare case with encephalopathy. Turk Pediatri Ars. 2016 Sep 1;51(3):169-172.

5. Domínguez-Carral J, Puertas-Martín V, Carreras-Sáez I, Maraña-Pérez Al, EscobarDelgado T, García-Peñas JJ. Neurological symptoms in children with intussusception. An Pediatr (Barc). 2014 May;80(5):293-8.
6. Hoisington G, Bartlett W, Kelly T. Intussusception presenting as encephalopathy. lowa Med. $1993 \mathrm{Mar} ; 83(3): 107-10$.

7. Godbole A, Concannon P, Glasson M. Intussusception presenting as profound lethargy. J Paediatr Child Health. 2000 Aug;36(4):392-4.

8. d'Escrienne MM, Velin P, Filippigh P, Steyaert $H$, Valla JS, Perelman S, Dupont D. [Lethargic form of acute intestinal intussusception in an infant]. Arch Pediatr. 1996 Jan;3(1):44-6.

9. Singer J. Altered consciousness as an early manifestation of intussusception. Pediatrics. 1979 Jul;64(1):93-5.

10. Ros SP, Reynolds SL, Bhisitkul DM, Ionides SP, Handa RJ. Plasma beta-endorphin levels and childhood intussusception. J Emerg Med. 1994 Nov-Dec;12(6):767-9.

11. Angel M], Young GB. Metabolic encephalopathies. Neurol Clin. 2011 Nov; $29(4): 837-82$.

\section{Acknowledgments}

I would like to thank Dr. Sasikumar M S, Head of Pediatric Surgery, for his guidance and supervision and Dr. Rajakumar, Professor of Pediatrics for his assistance in the completion of my case report.

Conflict of Interest Statement at Funding

The Authors have no funding, financial relationships or conflicts of interest to disclose.

Author Contributions

Conceptualization: VM. Validation: SM. Investigation: VM. Resources: SR. Writing - Original Draft: VM. Writing - Review 亿 Editing: SM. Visualization: VM.

Cite as:

Mampilly V, Sankaran SM, Subbiah R. Encephalopathy: An Atypical Presentation of Intussusception. A Case Report. Int J Med Students. 2020 Jan-Apr;8(1):4144.

This work is licensed under a Creative Commons Attribution 4.0 International License

ISSN 2076-6327

This journal is published by the University Library System, University of Pittsburgh as part of the Digital Publishing Program and is co-sponsored by the University of Pittsburgh Press. 